\title{
Tratamiento quirúrgico de la ruptura septal ventricular postinfarto
}

\author{
Surgical treatment of postinfarction \\ ventricular septal rupture \\ Silvia Hernández-Meneses, ${ }^{*}$ Luis Raúl Meza-López, ${ }^{\ddagger}$ \\ Jorge Tizoc Olvera-Lozano, ${ }^{*}$ Belinda Elizabeth González-Díaz ${ }^{\S}$
}

Palabras clave: Infarto agudo al miocardio, ruptura septal ventricular postinfarto,

procedimiento quirúrgico.

Keywords:

Myocardial infarction, ventricular septal rupture, surgical procedure.

\footnotetext{
* Departamento de Cirugía Cardiovascular. UMAE Hospital de Cardiología del Centro Médico Nacional Siglo XXI. IMSS. Ciudad de México.

¥ Departamento de Cirugía Cardiovascular. Hospital Regional de Alta Especialidad "Bicentenario de la Independencia” ISSSTE. Estado de México.
}

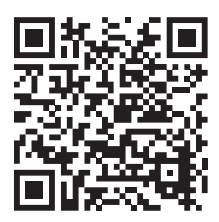

\section{RESUMEN}

Introducción: La ruptura postinfarto del septum ventricular es una complicación mecánica del infarto agudo al miocardio, con alta mortalidad; pero la evolución de las técnicas en quirófano ha llevado a su reparación temprana con mejores resultados. Objetivo: Describir la experiencia quirúrgica en la rotura septal ventricular postinfarto en pacientes del Hospital de Cardiología, Centro Médico Nacional Siglo XXI. Métodos: Estudio descriptivo, retrolectivo, observacional, serie de casos. Datos recabados de expedientes que cumplieron los criterios de inclusión. Resultados: Del $1^{\circ}$ de enero de 1999 al 30 de abril de 2015 se registraron 49 enfermos que fueron sometidos a restauración mediante cirugía, sólo 27 cumplieron con los criterios de inclusión: 15 hombres y 12 mujeres, edad promedio 62 años, mortalidad general $63 \%$. De los sobrevivientes, $50 \%$ presentaron defecto residual septal del ventrículo: un paciente fue llevado a reoperación para corrección total y el resto de los enfermos continúa en seguimiento. Conclusiones: Los mejores resultados quirúrgicos se obtuvieron en aquellos sujetados a reconstrucción de la rotura del septum ventricular postinfarto luego de dos semanas del diagnóstico del infarto agudo al miocardio, encontrándose en clase funcional I y II de la New York Heart Association. En cuanto a la sobrevida, los participantes que se sometieron a reparación con parche simple presentaron menor incidencia de desperfectos residuales.

\section{ABSTRACT}

Introduction: Postinfarction ventricular septal rupture is a high mortality complication of myocardial infarction; but the evolution of surgical techniques has led to early repair with better results. Objective: To describe the surgical experience in patients with postinfarction ventricular septal rupture at the Cardiology Hospital of CMN SXXI. Methods: A retrospective, observational case series study was conducted. Collected data records that met the inclusion criteria were considered. Results: From January first, 1999, to April 30, 2015, 49 patients who underwent surgical repair were registered, only 27 met the inclusion criteria: 15 men and 12 women. Mean age was 62 years old, with $63 \%$ overall mortality. Of surviving patients, $50 \%$ presented a residual ventricular septal defect: one patient underwent reoperation for total correction, and the remaining continued on follow up. Conclusions: The best surgical outcomes were obtained in patients undergoing repair after two weeks of diagnosis of myocardial infarction, being in functional class I and II of the New York Heart Association. As to survival, patients who underwent simple patch repair had lower incidence of residual defects.

\author{
Abreviaturas: \\ RSVP $=$ Ruptura septal ventricular postinfarto. \\ IAM = Infarto agudo al miocardio \\ $\mathrm{FRCV}=$ Factores de riesgo cardiovascular. \\ EUROSCORE $=$ European System for Cardiac Operative \\ Risk Evaluation. \\ $\mathrm{DCP}=$ Derivación cardiopulmonar.
}

PAo $=$ Pinzamiento aórtico.

$\mathrm{BIAC}=$ Balón intraaórtico de contrapulsación

$\mathrm{UCIC}=$ Unidad de cuidados intensivos coronarios.

$\mathrm{NYHA}=$ New York Heart Association.

$\mathrm{ECG}=$ Electrocardiograma.

$\mathrm{SICA}=$ Síndrome isquémico coronario agudo.

Citar como: Hernández-Meneses S, Meza-López LR, Olvera-Lozano JT, González-Díaz BE. Tratamiento quirúrgico de la ruptura septal ventricular postinfarto. Cir Gen. 2020; 42 (4): 281-287. https://dx.doi.org/10.35366/101397 
$\S$ Departamento de Hemodinamia. UMAE Hospital de Cardiología del Centro Médico Nacional Siglo XXI. IMSS. Ciudad de México.

UMAE Hospital de Cardiología. Centro Médico Nacional Siglo XXI. IMSS Ciudad de México.

Recibido: 23/08/2019 Aceptado: 30/05/2021

\section{INTRODUCCIÓN}

L as complicaciones mecánicas del infarto agudo al miocardio (IAM) constituyen uno de los más indeseables y dramáticos contratiempos que pueden presentarse. ${ }^{1} \mathrm{~A}$ pesar de su baja incidencia $(<1 \%),{ }^{2}$ la gravedad que implican hace preciso un diagnóstico rápido y acertado, así como tratamiento oportuno. Se presentan como tres entidades bien definidas: la ruptura del septum ventricular, las roturas de la pared libre del ventrículo y del músculo papilar con insuficiencia mitral. ${ }^{1}$

La rotura septal ventricular postinfarto (RSVP) ocurre con mayor frecuencia durante la primera semana posterior a un IAM, típicamente de tres a cinco días después. ${ }^{2}$ El uso de agentes trombolíticos redujo su incidencia, de $1-2 \%$ en la era pretrombolítica, a sólo $0.2 \%$ en la actualidad. Sin embargo, a pesar de la atención veloz y efectiva en la era de reperfusión coronaria (trombólisis mecánica y química), se ha reportado una mortalidad de $73.8-87 \%$, considerándose una cifra aproximada de $45 \%$ para los pacientes tratados quirúrgicamente, y de $90 \%$ entre aquellos sometidos únicamente a tratamiento médico. ${ }^{3-5}$

Debido a los pobres resultados del procedimiento médico, la intervención con cirugía resulta necesaria, ${ }^{5}$ sin embargo, la operación urgente también se relaciona con fragilidad tisular y cortocircuito residual. La evolución de las técnicas en el quirófano ${ }^{2}$ ha llevado a la reparación temprana y ha disminuido la mortalidad de $90 \%$ a aproximadamente un $50 \%{ }^{3}$

Los enfermos que se encuentran completamente estables, sin apoyo de dispositivos de asistencia ventricular, representan $5 \%$ o menos de los casos de RSVP, y en ellos se puede retrasar la cirugía con buenos resultados. ${ }^{6}$ Por el contrario, posponer el procedimiento en aquellos con choque cardiogénico representa un manejo fallido. Los dolientes que se encuentran en una posición intermedia entre el choque cardiogénico y la estabilidad hemodinámica deben ser intervenidos a la brevedad (12-24 horas) luego de una evaluación preoperatoria adecuada. Para muchos pacientes con descompensación aguda, las recientes mejoras en técnicas quirúrgicas han permitido realizar cirugías tempranas reduciendo el riesgo de deterioro

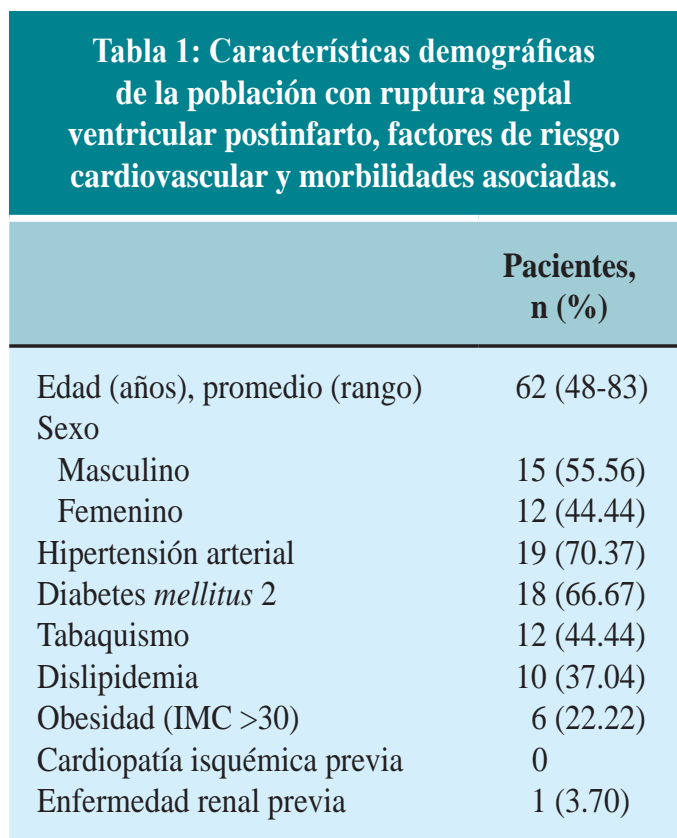

IMC = índice de masa corporal.

hemodinámico, a costa de un incremento en la dificultad técnica. ${ }^{2,7,8}$

Debido a la complejidad de esta patología, se han desarrollado diferentes abordajes con la finalidad de mejorar los resultados de las cirugías y la supervivencia. Dentro de las técnicas quirúrgicas conocidas se puede mencionar a la infartectomía con cierre, la exclusión del infarto, el uso de pegamento biológico, el cierre con doble parche, la exclusión septal, cierre mediante técnica de Sándwich y el cierre con parche simple. Por otro lado, el desarrollo de técnicas percutáneas como el uso de un dispositivo Amplatzer ha permitido, en casos seleccionados, el cierre percutáneo de los defectos del septum. ${ }^{2,8}$

El objetivo de esta investigación fue describir las características generales de los enfermos diagnosticados con RSVP y los resultados obtenidos tras la restauración mediante cirugía, evaluando la mortalidad, complicaciones posoperatorias y la supervivencia.

\section{MÉTODOS}

Se llevó a cabo un estudio descriptivo, observacional, retrolectivo, del tipo serie de casos 
consecutivos, en el que se incluyó a todos los internados registrados desde enero de 1999 hasta abril de 2015, quienes se sujetaron a

\begin{tabular}{|c|c|}
\hline & $\begin{array}{c}\text { Pacientes, } \\
\text { n (\%) }\end{array}$ \\
\hline \multicolumn{2}{|l|}{ Tipo de infarto } \\
\hline CEST & $26(96.30)$ \\
\hline SEST & $1(3.70)$ \\
\hline \multicolumn{2}{|l|}{ Localización del infarto } \\
\hline Anterior & 18 (66.67) \\
\hline Inferior & $9(33.33)$ \\
\hline \multicolumn{2}{|l|}{ Tratamiento al ingreso hospitalario } \\
\hline Conservador & 23 (85.19) \\
\hline Trombólisis & $3(11.11)$ \\
\hline ICP & $1(3.70)$ \\
\hline \multicolumn{2}{|l|}{ Lesiones coronarias } \\
\hline Enfermedad de un vaso principal & $13(48.15)$ \\
\hline DA & 7 (53.85) \\
\hline $\mathrm{CD}$ & $4(30.77)$ \\
\hline $\mathrm{CX}$ & $2(15.38)$ \\
\hline Enfermedad de dos vasos & $5(18.52)$ \\
\hline Enfermedad trivascular & $4(14.81)$ \\
\hline EAMV & $5(18.52)$ \\
\hline \multicolumn{2}{|l|}{ No. cortocircuitos } \\
\hline Simple & $27(100)$ \\
\hline Apical & $7(25.93)$ \\
\hline Anterior & $12(44.44)$ \\
\hline Posterior & $6(22.22)$ \\
\hline Segmento medio & $2(7.41)$ \\
\hline Complejos & 0 \\
\hline \multicolumn{2}{|l|}{ FEVI $(\%)$} \\
\hline$<30$ & $5(18.52)$ \\
\hline $31-50$ & $16(59.26)$ \\
\hline$>50$ & $6(22.22)$ \\
\hline $\begin{array}{l}\text { Complicaciones asociadas a RSVP } \\
\text { Choque cardiogénico }\end{array}$ & $15(55.56)$ \\
\hline Arritmias & $3(11.11)$ \\
\hline Taquicardia ventricular & $1(33.33)$ \\
\hline BAV & $2(66.67)$ \\
\hline
\end{tabular}

CEST = con elevación del segmento ST, SEST = sin elevación del segmento ST, ICP = intervencionismo coronario percutáneo, DA = descendente anterior, DX $=$ diagonal, $\mathrm{CX}=$ circunfleja, $\mathrm{EAMV}=$ enfermedad ateromatosa de múltiples vasos, FEVI = fracción de eyección del ventrículo izquierdo, RSVP = ruptura septal ventricular postinfarto, BAV = bloqueo auriculoventricular, EAMV = enfermedad ateromatosa de múltiples vasos. cirugía de reparación de RSVP y que contaran con expedientes completos, en el Hospital de Cardiología del Centro Médico Nacional Siglo XXI del Instituto Mexicano del Seguro Social.

Para el manejo analítico de los datos se utilizó estadística descriptiva, medidas de tendencia central $y \chi^{2}$ a fin de examinar los factores relacionados a la mortalidad.

\section{RESULTADOS}

Del primero de enero de 1999 al 30 de abril de 2015 se registró un total de 49 enfermos con diagnóstico de RSVP, que fueron sometidos a reconstrucción quirúrgica en el servicio de $\mathrm{Ci}$ rugía Cardiovascular, pero sólo 27 cumplieron con los criterios de inclusión.

Los datos demográficos de la población, los factores de riesgo cardiovascular (FRCV) y las morbilidades asociadas se resumen en la Tabla 1. Las características clínicas de los participantes se presentan en la Tabla 2.

El indicador EUROSCORE (European System for Cardiac Operative Risk Evaluation) promedio fue de $53.93 \%$, con un rango de 16.13-91.41\%. Todos los aquejados fueron hospitalizados en la Unidad de Cuidados Intensivos Coronarios (UCIC) como parte del manejo del IAM; el manejo otorgado durante su estancia en dicho servicio y el estado clínico de dichos enfermos son presentados en la Tabla 3.

En cuanto a la técnica de quirófano que se utilizó, en 21 pacientes (77.78\%) la reparación fue realizada mediante cierre con parche simple, mientras que seis casos (22.22\%) fueron con parche doble. El resto de los procedimientos concomitantes aplicados y los materiales empleados se indican en la Tabla 4.

En minutos, el tiempo promedio de derivación cardiopulmonar (DCP) fue de 135 (rango 62-313) con un lapso promedio de pinzamiento aórtico (PAo) de 90 (rango 49-153).

Las contratiempos posquirúrgicos reportados fueron: sangrado mayor al habitual en cinco enfermos (18.52\%), choque cardiogénico en 16 del grupo (59.26\%), falla renal aguda en 10 de ellos (37.04\%), infarto perioperatorio en un caso $(3.70 \%)$, acidosis metabólica refractaria a tratamiento en otros cinco (18.52\%), arritmias en dos pacientes $(7.40 \%)$ (un paciente con taquicardia ventricular y uno con bloqueo au- 
Tabla 3: Tratamiento perioperatorio de los pacientes con diagnóstico de ruptura septal ventricular postinfarto.

\begin{tabular}{lc} 
& $\begin{array}{c}\text { Pacientes, } \\
\mathbf{n}(\%)\end{array}$ \\
& $16(59.26)$ \\
Uso de inotrópicos & $14(51.85)$ \\
Uso de BIAC & \\
Intervencionismo & $1(3.70)$ \\
Amplatzer & $3(11.11)$ \\
ICP & $2(66.67)$ \\
$\quad$ Exitosa & $1(33.33)$ \\
$\quad$ Fallida & \\
Clase funcional de la NYHA & $1(3.70)$ \\
I & $10(37.04)$ \\
II & $4(14.82)$ \\
III & $12(44.44)$ \\
IV & \\
Tiempo diagnóstico IAM-tratamiento & \\
quirúrgico RSVP (días) & \\
< 7 -15 & $12(44.44)$ \\
$>15$ & $11(40.74)$ \\
\hline
\end{tabular}

BIAC = balón intraaórtico de contrapulsación, ICP = intervencionismo coronario percutáneo, NYHA = New York Heart Association, IAM = infarto agudo al miocardio, RSVP = ruptura septal ventricular postinfarto. para la reparación del desperfecto y procedimientos concomitantes realizados durante la cirugía (Tabla 6).

De los 10 sobrevivientes, ocho aún continúan en seguimiento, y de estos últimos, cuatro persisten con deterioro septal interventricular residual sin repercusión hemodinámica que amerite cierre por intervencionismo o quirúrgico, manteniéndose clínicamente en clase funcional II de la NYHA.

De aquellos pacientes que sobrevivieron sin defecto residual y que continúan en seguimiento, el $75 \%$ fueron sometidos a cierre con parche simple y revascularización concomitante, y solamente en un paciente se realizó restauración con parche doble. En cuanto a los enfermos con daño residual, el 50\% fue reconstruido con doble parche, y uno de ellos fue llevado a reoperación persiguiendo el cierre del defecto residual; el tamaño del desarreglo reportado en los expedientes es de 11, 9 y 3 milímetros, que como se mencionó anteriormente, en el seguimiento por la consulta externa no ameritaron tratamiento.

\section{DISCUSIÓN}

La RSVP es una complicación rara pero letal del IAM. ${ }^{1-4,7,9,10}$ Usualmente produce un gran cortocircuito de izquierda a derecha que deteriora el estado clínico de los enfermos. ${ }^{1,3,7,11}$ Respecto a las características demográficas de la población, en la presente investigación ${ }^{1,11}$ no se encontró correlación con lo reportado en la literatura respecto al sexo; ya que prácticamente se observó una relación hombre:mujer de 1:1.

Los factores de riesgo cardiovascular más frecuentemente asociados en este estudio fueron diabetes mellitus, hipertensión y dislipidemia. ${ }^{1,11}$ En ninguno de los casos se encontró antecedente de cardiopatía isquémica previa que, como comentan Caballero Borrego y colaboradores, es un factor protector para el desarrollo de RSVP en los afectados por IAM, debido al desarrollo de circulación colateral.

Predominantemente se encontró síndrome isquémico coronario agudo con elevación del segmento ST (SICA CEST), y por electrocardiografía (ECG) la localización más 
frecuente del infarto fue en la cara anterior. La evaluación angiográfica revela que, por lo general, la RSVP se asocia a oclusión de una arteria coronaria principal y estos pacientes tienen menor desarrollo de vasos colaterales. Sobre los hallazgos angiográficos de la población, especialmente se observó afección de un vaso principal (casi en un 50\% de los casos), y de éstos, la arteria más comúnmente afectada fue la descendente anterior. Esto igualmente se correlaciona con lo reportado en la literatura. ${ }^{11,12}$

Con el inicio de la terapia de reperfusión urgente para el IAM (incluyendo trombólisis e intervención coronaria percutánea) disminuyó la incidencia de RSVP, al restaurarse el flujo de la arteria relacionada con el infarto, buscando salvar el miocardio y reducir la incidencia de infarto transmural. ${ }^{4,10,11}$ En este reporte, en la mayor parte de los enfermos (85\%) no se dio tratamiento de reperfusión coronaria.

La localización anatómica del defecto fue anteroapical en aproximadamente $60 \%$ de los casos, y de 20 a $40 \%$ en el tabique posterior. En este trabajo, todos los pacientes presentaron deterioros septales ventriculares simples, descritos por ecocardiografía, lo cual se corroboró con los hallazgos quirúrgicos reportados, y la ubicación más común fue en el segmento an-

Tabla 4: Manejo quirúrgico empleado.

\begin{tabular}{lc} 
Tratamiento quirúrgico & $\begin{array}{c}\text { Pacientes, } \\
\mathbf{n}(\%)\end{array}$ \\
\hline Reparación con parche simple & $21(77.78)$ \\
Reparación con parche doble & $6(22.22)$ \\
Cierre + exclusión infarto & $2(7.41)$ \\
Cierre + revascularización miocárdica & $13(48.15)$ \\
Cierre + DAV & $1(3.70)$ \\
Cierre + plastia mitral & $1(3.70)$ \\
Tipo de material empleado & \\
Pericardio bovino & $12(44.44)$ \\
Pericardio autólogo & $1(3.70)$ \\
Poliéster & $5(18.52)$ \\
Teflón & $3(11.11)$ \\
Dacrón & $3(11.11)$ \\
PTFE & $3(11.11)$ \\
\hline
\end{tabular}

$\mathrm{DAV}=$ dispositivo de asistencia ventricular, $\mathrm{PTFE}=$ politetrafluoroetileno. terior, lo que correspondió a la lesión coronaria más frecuentemente asociada al IAM. ${ }^{11,12}$

Llama la atención que casi en el $80 \%$ de los casos se encontró preservado el funcionamiento del ventrículo izquierdo, con una FEVI > $30 \%$ y, como se halló en las complicaciones de la RSVP, más de un 50\% de los casos presentó choque cardiogénico franco, por lo tanto, no se correlacionaron estos factores. La función ventricular depende de la severidad del infarto, con el subsecuente aturdimiento miocárdico y las modificaciones hemodinámicas generadas por el cortocircuito de izquierda a derecha, que ocasionan falla en el ventrículo derecho con riesgo de daño biventricular. ${ }^{1,2,7,11}$

Las complicaciones más frecuentemente observadas como consecuencia de la RSVP fueron: el choque cardiogénico (60\%), y en un $11 \%$ las arritmias del tipo taquicardia ventricular y bloqueo auriculoventricular completo.

Como consecuencia de las complicaciones y de la inestabilidad hemodinámica de los incluidos en el estudio, la mayoría ameritó manejo con inotrópicos, así como apoyo circulatorio con BIAC en el preoperatorio. Y corroborando la gravedad de la enfermedad, motivo de este trabajo, el EUROSCORE II promedio calculado para los enfermos, fue mayor al $50 \%$. $^{2,4,7,8}$

En cuanto al manejo con cirugía realizado en este hospital, en aproximadamente el $85 \%$ de los casos, este se llevó a cabo dentro de los primeros 15 días posteriores al diagnóstico del IAM, y la técnica quirúrgica empleada con mayor frecuencia intentando la reparación del defecto del septum fue la colocación de parche simple de pericardio bovino. Los tiempos de DCP y PAo promedio fueron de 135 y 90 minutos respectivamente.

Las complicaciones más comunes en el posquirúrgico inmediato fueron el choque cardiogénico persistente (59\%), la falla renal aguda $(37 \%)$, y finalmente las infecciones en un 33\% de los casos (neumonía, mediastinitis e infección de vías urinarias).

En esta investigación, la mortalidad de las personas se correlacionó con la reportada a nivel mundial, representada por un $63 \%$. $3-5,7,8,10-$ ${ }^{13}$ Los factores analizados y asociados con una significancia estadística a tal consecuencia, fueron la clase funcional IV de la NYHA, y el lapso en el cual habían sido sometidos a recons- 
Tabla 5: Mortalidad asociada al tratamiento preoperatorio y estado clínico de los pacientes diagnosticados con ruptura septal ventricular postinfarto.

\begin{tabular}{lccc} 
& $\begin{array}{c}\text { Mortalidad Sobrevida } \\
\text { (pacientes) }\end{array}$ & (pacientes) & $\mathbf{p}^{*}$ \\
& 12 & 4 & NS \\
\hline Uso de inotrópicos & 11 & 3 & NS \\
Uso de BIAC & & & \\
Intervencionismo & 2 & 1 & NS \\
$\quad$ ICP & 1 & 0 & \\
$\quad$ Amplatzer & & & 0.01 \\
Clase funcional & 4 & 7 & \\
$\quad$ I/II & 13 & 3 & \\
$\quad$ III/IV & & & \\
Tiempo entre diagnóstico IAM-cirugía (días) & 11 & 1 & 0.012 \\
$\quad<7$ & 6 & 5 & \\
$7-15$ & 0 & 4 & \\
$>15$ & & &
\end{tabular}

${ }^{*} \mathrm{p}$ = prueba de $\chi^{2}$, BIAC = balón intraaórtico de contrapulsación,

ICP = intervencionismo coronario percutáneo, IAM = infarto agudo al miocardio,

$\mathrm{NS}=$ no significativa

\section{Tabla 6: Análisis de la mortalidad del tratamiento quirúrgico.}

\begin{tabular}{lccc} 
& $\begin{array}{c}\text { Mortalidad } \\
\text { (pacientes) }\end{array}$ & $\begin{array}{c}\text { Sobrevida } \\
\text { (pacientes) }\end{array}$ & $\mathbf{p}^{*}$ \\
\hline Técnica para reparación del defecto & & & \\
$\quad$ Parche simple & 14 & 7 & NS \\
Parche doble & 3 & 3 & \\
Procedimientos concomitantes & & & NS \\
RVM & 8 & 5 & \\
Exclusión de infarto & 1 & 1 & \\
Plastia M & 0 & 1 & \\
DAV & 1 & 0 & \\
\hline
\end{tabular}

*p = prueba de $\chi^{2}, \mathrm{RVM}=$ revascularización miocárdica, $\mathrm{M}$ = mitral,

$\mathrm{DAV}=$ dispositivo de asistencia ventricular, NS = no significativa.

trucción del daño septal ventricular posterior al IAM, ya que en aquellos pacientes en quienes fue retrasado el procedimiento más de 15 días, la sobrevida fue del $100 \%$ y, como se encuentra en la literatura, entre los determinantes de ese desenlace de la cirugía se encuentran el tiempo entre el IAM y la reparación del defecto, el choque cardiogénico, la disfunción ventricular izquierda, falla renal y enfermedad coronaria previa. $5,7,11,13-15$

Actualmente se sugiere que, de ser posible, debe retrasarse el manejo quirúrgico hasta que el paciente cumpla por lo menos tres semanas, con objeto de delimitar mejor el área de necrosis, aunque es claro que en la mayoría de los enfermos no se puede posponer el tratamiento debido a la inestabilidad hemodinámica que condiciona el cortocircuito de izquierda a derecha; además de que, en los afectados que sólo reciben atención médica, la mortalidad es prácticamente del 100\%. 4,7,8,11,13

A pesar de que evitar los cortocircuitos residuales y minimizar el daño de áreas no infartadas son los objetivos más importantes en el manejo de la RSVP, el cortocircuito residual continúa siendo un problema mayor en los enfermos. El factor más importante que incide en la fuga después de la reconstrucción de roturas septales posteriores es la dificultad técnica en identificar el desperfecto en el septum, particularmente al suturar tejido muscular necrótico. Los defectos residuales pueden ocurrir por deficiente cobertura del daño o desgarro del miocardio friable de la línea de sutura. ${ }^{15}$

En el seguimiento de los afectados que sobrevivieron, se observó que aproximadamente la mitad presentó deterioro septal ventricular residual. Cabe resaltar que, en dichos pacientes, un $50 \%$ fue sometido a reparación del daño con parche simple, mientras que el resto fue restaurado con doble parche. Sin embargo, no existió diferencia estadísticamente significativa para poder determinar si alguna técnica influyó en la presencia de lesión residual.

De aquellos pacientes que sobrevivieron, y en los cuales no se documentó defecto septal ventricular residual, se observa que $75 \%$ fueron sujetados a reparación con parche simple y revascularización concomitante. Igualmente, no se encontró diferencia estadísticamente significativa en estos resultados, pero se podría establecer que, clínicamente, esto puede deberse a la mayor experiencia de los cirujanos de este hospital con dicha técnica, más que a ventajas que pudiera ofrecer la misma con respecto a otras.

Debido a las limitaciones en cuanto al diseño de este análisis no resulta posible emitir más 
relaciones entre las variables; sin embargo, los autores consideran que es de gran utilidad para conocer la experiencia quirúrgica en el manejo de esta complicación del IAM.

\section{CONCLUSIONES}

La RSVP es una complicación letal del IAM, cuyo manejo deviene todo un reto y que implica un abordaje integral por parte de un equipo multidisciplinario. La actuación en quirófano constituye una opción viable para estos pacientes. Los mejores resultados se observaron en aquellos enfermos sometidos a cirugía luego de dos semanas del diagnóstico del IAM, y en esos otros que se encontraban en CF I y II de la NYHA antes de ser intervenidos en un procedimiento quirúrgico.

Debido a las limitaciones de esta investigación, se considera además que en este medio resulta necesario realizar más estudios al respecto, con la finalidad de ampliar la experiencia en el tratamiento de esta patología.

\section{REFERENCIAS}

1. Caballero-Borrego J, Hernández-García JM, SanchisFores J. Complicaciones mecánicas en el infarto agudo al miocardio. ¿Cuáles son, cuál es su tratamiento y qué papel tiene el intervencionismo percutáneo? Rev Esp Cardiol Supl. 2009; 9: 62C-70C.

2. Isoda S, Osako M, Kimura T, Nishimura K, Yamanaka N, Nakamura N, et al. Surgical repair of postinfarction ventricular septal defects 2013 Update. Ann Thorac Cardiovasc Surg. 2013; 19: 95-102.

3. Siondalski P, Jarmoszewics K, Rogowski J, Jurowiecki J. Emergency surgical closure of postinfarction ventricular septal defect on the beating heart. Interact Cardiovasc Thorac Surg. 2007; 6: 160-162.

4. Barros de Oliviera MP, Barros de Oliveira MV, Barbosa $\mathrm{CH}$, de Carvalho Silva NP, Renda de Escobar R, Gonçalves de Rueda F, et al. Clinical and surgical profile of patients operated for postinfarction ventricular septal rupture. Rev Bras Cir Cardiovasc. 2010; 25 (3): 341-349.

5. Rohn V, Grus T, Lindner J, Lips M, Belohlavek J. Postinfarction ventricular septal rupture: a rare complication remains challenge for cardiac surgical team. Prague Medical Report. 2013; 114: 9-17.

6. Swinkels BM, Peters RHJ, Van den Brink A. Successful management of postinfarction ventricular septal rupture. Neth Heart J. 2005; 13: 18-20.

7. Grandmougin D, Fayad G, Delolme MC, Metton O, Vola M, Azzaoui R, et al. Beating-heart approach and moderately delayed surgical management of postinfarction ventricular septal rupture: an advisable approach to improve results? Interact Cardiovasc Thoracic Surg. 2005; 4: 238-241.

8. Gregoric ID, Bieniarz MC, Arora $\mathrm{H}$, Frazier $\mathrm{OH}$, Kar B, Loyalka P. Percutaneous ventricular assist device support in a patient with a postinfarction ventricular septal defect. Tex Heart Inst J. 2008; 35: 46-49.

9. Xiao-Ying $\mathrm{H}$, Hong Q, Shu-bin Q, Lian-ming K, Lei $\mathrm{S}$, Jun Z, et al. Clinical analysis and stratification of ventricular septal rupture following acute myocardial infarction. Chin Med J. 2013; 126: 4105-4108.

10. Arnaoutakis GJ, Zhao Y, George TJ, Sciortino CM, McCarthy PM, Conte JV. Surgical repair of ventricular septal defect myocardial infarction: outcomes from the society of thoracic surgeons national database. Ann Thorac Surg. 2012; 94: 436-444.

11. Jones BM, Kapadia SR, Smedira MG, Robich M, Tuzcu EM, Menon $\mathrm{V}$, et al. Ventricular septal rupture complicating acute myocardial infarction: a contemporary review. Eur Heart J. 2014; 35: 2060-2068.

12. Espinosa-Ledesma A, Ramírez-Orozco F, HerreraCamacho G, Macías-Amezcua MD, González-Ojeda A, Fuentes Orozco C. Mortalidad de la ruptura de septum interventricular posterior a infarto agudo al miocardio con manejo quirúrgico. Cir Cir. 2012; 80: 496-503.

13. Rojas Velasco G, Lerma C, Arias Mendoza A, Alvarez Sangabriel A, Altamirano A, Azar Manzur F, et al. Características clínicas, modalidades de tratamiento y mortalidad en la ruptura del septum interventricular postinfarto. Arch Cardiol Mex. 2011; 81: 197-203.

14. Costache VS, Chavanon O, Bouvaist H, Blin D. Early Amplatzer occluder closure of a postinfarct ventricular septal defect as a bridge to surgical procedure. Interact Cardiovasc Thorac Surg. 2007; 6: 503-504.

15. Balkanay M, Eren E, Keles E, Toker ME, Guler M. Double-patch repair of postinfarction ventricular septal defect. Tex Heart Inst J. 2005; 32: 43-46.

\section{Consideraciones y responsabilidad ética:} Debido a la naturaleza del estudio (únicamente revisión de los expedientes clínicos) no se requirió de consentimiento informado, ni aprobación por el comité de ética en investigación.

Financiamiento: No se recibió apoyo financiero para la realización de esta investigación.

Conflicto de intereses: Ninguno.

Correspondencia:

Silvia Hernández-Meneses

Avenida Cuauhtémoc Núm. 330,

Col. Doctores, 06720,

Alcaldía Cuauhtémoc,

Ciudad de México, México,

E-mail:shm286@hotmail.com 